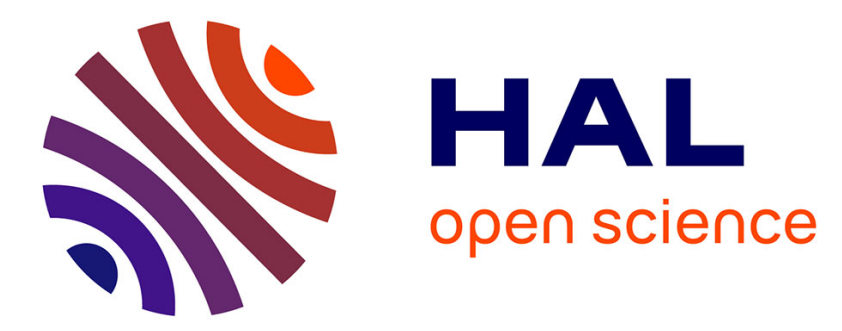

\title{
Self-Sensing method considering the dynamic impedance of piezoelectric based actuators for ultra-low frequency
} Joël Bafumba Liseli, Joel Agnus, Philippe Lutz, Micky Rakotondrabe

\section{To cite this version:}

Joël Bafumba Liseli, Joel Agnus, Philippe Lutz, Micky Rakotondrabe. Self-Sensing method considering the dynamic impedance of piezoelectric based actuators for ultra-low frequency. IEEE Robotics and Automation Letters, 2018, 3 (2), pp.1049 - 1055. 10.1109/LRA.2018.2794514 . hal-02399257

\section{HAL Id: hal-02399257 \\ https://hal.science/hal-02399257}

Submitted on 9 Dec 2019

HAL is a multi-disciplinary open access archive for the deposit and dissemination of scientific research documents, whether they are published or not. The documents may come from teaching and research institutions in France or abroad, or from public or private research centers.
L'archive ouverte pluridisciplinaire $\mathbf{H A L}$, est destinée au dépôt et à la diffusion de documents scientifiques de niveau recherche, publiés ou non, émanant des établissements d'enseignement et de recherche français ou étrangers, des laboratoires publics ou privés. 


\title{
Self-Sensing method considering the dynamic impedance of piezoelectric based actuators for ultra-low frequency
}

\author{
Joël BAFUMBA LISELI, Joël AGNUS, Philippe LUTZ , Member, IEEE, and Micky \\ RAKOTONDRABE, Member, IEEE,
}

\begin{abstract}
This paper presents a displacement self-sensing method for piezoelectric actuators that takes into account the effect of the deformation of these latter on their electrical impedance. A self-updating digital electrical impedance equivalent to that of the piezoelement is suggested in order to overcome the mismatch problem met when considering a static analog electrical impedance as classically used. Then, the design of an observer that performs a thorough separation of the electric current due to the direct piezoelectric effect from the measured electric current is proposed in order to estimate the piezoelectric actuator displacement. The experimental results validate the proposed approach and demonstrate the efficiency of the developed self-sensing circuit and its observer to estimate the displacement of the piezoceramics actuator for long duration measurement of constant step signal.
\end{abstract}

Index Terms-Automation at Micro-Nano Scales, Micro/Nano Robots, Motion Control.

\section{INTRODUCTION}

$\mathbf{M}$ ICROASSEMBLY and micromanipulation applications require precise positioning with very high accuracy at micro/nanoscale and a control of the applied force on the manipulated object due, among other causes, to the fragility of objects at this scale. Piezoelectric materials are very prized to develop systems acting at this scale. This recognition is thanks to the high resolution, high bandwidth and high force density they can offer. Despite the high resolution and the high speed of these materials, nonlinearities such as hysteresis and creep strongly compromise the accuracy [1]. Feedback control can be used to remove the hysteresis and creep effects. However, applications such as precise positioning suffer from the lack of embeddable and precise sensors, both for displacement and force, to be used for packaged and feedback controlled systems. As an alternative to feedback control, open-loop, also called feedforward, control techniques can be used [2, 3, 4]. Even though this sensorless control approach gets rid of the need of expensive and bulky sensors to control the positioning

Manuscript received: September, 8, 2017; Revised November, 28, 2017; Accepted December, 28, 2017.

This paper was recommended for publication by Editor Yu Sun upon evaluation of the Associate Editor and Reviewers' comments.

This work has been supported by the Labex ACTION project (contract "ANR-11-LABX-0001-01") and the Bourgogne Franche-Comté region.

The authors are with the Department of Automatic Control and Micro-Mechatronic Systems, FEMTO-ST Institute, Univ. Bourgogne Franche-Comté, CNRS, 24 rue Savary, F-25000 Besançon, France. (jbafumbalfemto-st.fr, joel.agnus@femto-st.fr, philippe.lutz@femto-st.fr, mratoton@femto-st.fr)

Digital Object Identifier (DOI): see top of this page. and the applied force, it does not take into account the external disturbances nor the internal uncertainties of the actuators models. The lack of robustness against external disturbances and uncertainties in the model are likely to induce loss in accuracy during the positioning.

Another alternative solution that combines the advantages of the feedback control (robustness) and feedforward control (embeddability) is the Self-Sensing approach $[5,6,7]$. In a piezoelectric actuator, the self-sensing approach permits to measure the signals resulting from the deformation of the piezoelectric actuator as a mean to extract the needed information to enable a feedback control. Self-sensing technique allows a single piece of piezoelectric material to concurrently sense and actuate in a closed loop system. One benefit of a self-sensing actuator is that the sensor and actuator are truly collocated. Collocated control has been shown to have a number of advantages relating to the closed loop stability of the structure [8]. Piezoelectric materials have become increasingly important as sensors and actuators in structural control applications $[9,10]$. A key characteristic of these materials is the use of the converse piezoelectric effect to actuate the structure in addition to the direct effect to sense structural deformation. Indeed, mechanical stress provokes the apparition of electrical charges on the material's surface (direct effect) and an electric field provokes the deformation of the material (converse effect). Due to the converse and direct effects, the electrodes used to supply the piezoelectric actuators can also be used to get the appearing charges.

Piezoelectric self-sensing allows actuating a piezoelectric device while measuring its displacement (strain) and/or applied force (stress) by using itself as its proper sensor. As aforementioned, a common drawback of self-sensing approaches in microelectromechanical systems (MEMS) is the fact that drive and sense electrodes share a common node resulting in a potentially large feedthrough path from actuation to sensing. This feedthrough originating from the piezoelectric actuator electrical impedance, if not properly accounted for, can almost entirely conceal the signal generated by the motion of the structure and is especially dominant if the same transduction principle is used for both actuation and sensing.

There are several self-sensing schematics depending on the application. All of those schematics are based on a dual feedthrough cancellation to recover the signal arising from the motion of the structure that is heavily buried in the feedthrough generated by the piezoelectric actuator electrical impedance. 
Bridge circuits $[7,11,12,13]$ are often used for strain rate sensing and are effective in suppressing the vibration modes but are not easy to balance for long-term measurements. In [14] an antiparallel compensation for the feedthrough with an integrator amplifier to enable a long-term measurement was introduced. In these works, an electrical equivalent impedance of a piezoelectric actuator was used in a bridge or in an antiparallel schematic to compensate the electrical behavior of the piezoelectric actuator. This compensation allowed the measurement of the charges induced by the piezoelectric actuator deformation.

The electrical characteristics of the piezoelectric ceramics is essential in the design and analysis of the self-sensing circuit. Several researches have been carried out on the design of equivalent circuit models of piezoelectric materials. Goldfarb et al [15] used a Maxwell resistive capacitance to represent the electrical hysteresis between voltage and charges while considering the energy dissipation. Park [16] found that the circuit model with a capacitor and internal resistor in series could represent the behavior of piezoelectric ceramics much better than the model with capacitor and resistor in parallel when considering the energy dissipation. Mingjie et al [17] combined the behavior of the parallel model at low frequency and that of the series model at high frequency and showed that this representation well fitted with experimental tests.

However, due to the electromechanical coupling nature of piezoelectric materials, the mechanical deformation of the piezoelectric elements greatly affects the electrical impedance and the circuit modeling. In other words, due to the converse piezoelectric effect, the application of an electric field $E$ (resulting from the applied voltage $V_{i n}$ ) creates a mechanical deformation in the piezoelectric actuator's crystals and with this deformation, the piezoelectric ceramics maintains its volume but does not keep the same proportions due to Poisson effect. Therefore, an equivalent electrical model with static values would not accurately depict the electrical characteristics of piezoelectric materials. Measurements using an impedance analyzer (Keysight E4990A) proved that the impedance of a piezoelectric material is dependent on the input voltage $V_{i n}$ and on the mechanical structure to which it is bonded (Figure 1). In the frequency response of Figure 1, one can notice that the impedance of a capacitor (reactance) dominates the electric impedance of the PZT actuator even though its amplitude varies with the applied electric field. Thus, it is accurate to presume that, for an equivalent electrical model of a PZT actuator, the capacitor is the element that has its value modified the most. Furthermore, the use of an analog integrator, as suggested by [14], permits to maintain the information about the charges that have been produced during the actuator deformation and tackles the leaking current problem. Therefore, an equivalent electrical model depicted by just an electric field dependent capacitor is sufficient for the design of a force and deformation measurement system using self-sensing propriety of piezoelectric materials.

The dynamic behavior of the piezoelectric implies that considering only a static equivalent model in a self-sensing circuit will unquestionably produce errors in the measurements of the charges generated by the piezoelement deformation $Q_{\delta}$
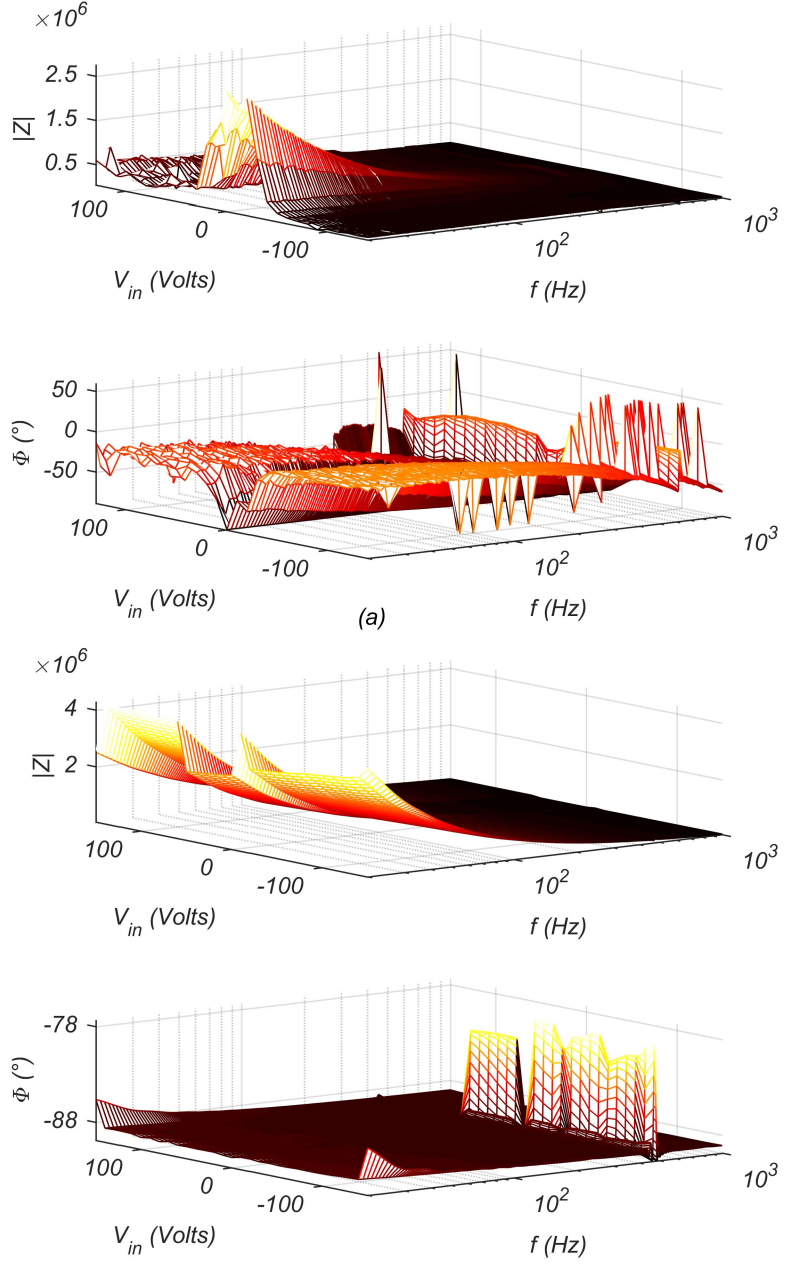

(b)

Fig. 1. Magnitude $|Z|$ and phase $\Phi$ of a bilayer unimorph as function of $V_{\text {in }}$ and $f$ (a) $200 \mu \mathrm{m}$ of PZT and $70 \mu \mathrm{m}$ of $\mathrm{Ni}$ (b) $200 \mu \mathrm{m}$ of PZT and $50 \mu \mathrm{m}$ of $\mathrm{Cu}$

and consequently, errors in the estimation of the displacement (strain) and/or the applied force. Unfortunately, no analog electrical component fits to the piezoelectric dynamic impedance. Nevertheless, a numerical equivalent electrical circuit can easily be shaped to tackle this issue. The benefit of a numerical processing is that it allows the use of more complex electrical impedance models of piezoelectric actuator.

We propose in this paper a way to reduce errors in the estimation of the displacement by considering the dynamic impedance of the piezoelectric actuator during the feedthrough cancelation.

The subsequent parts of this paper are organized as follows. Section II shows the electronic circuit and numerical treatment used for the static impedance cancellation. Section III presents the new observer that will include the dynamic characteristics for a more accurate measurement of $Q_{\delta}$. Finally, section IV and $\mathrm{V}$ will present the efficiency of the adopted approach and list the perspectives for the upcoming works. 


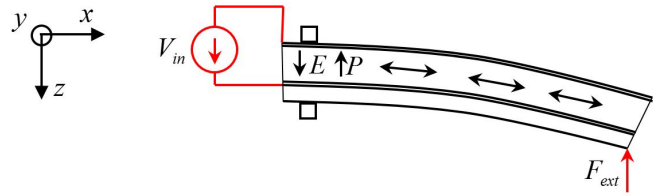

Fig. 2. A Bilayer unimorph piezoelectric cantilever beam during extension of the piezoelectric material.

\section{THE PROPOSED SIGNAL CONDITIONING}

Piezoelectric actuators or sensors are electromechanically coupled systems. For sensor applications, the mechanical effect on electrical parameters is described by the direct piezoelectric effect. Simple passive sensors output charges depending on the mechanical stress $T$ (or strain $S$ ). In a coupled system, however, the electric field $\mathrm{E}$ is an input parameter enforced by the electrical driving unit. For a defined $E$ in a piezoelectric system, the output charges on the electrodes $Q$ is dependent on both the mechanical and electrical inputs. The 1987, IEEE [18] proposed the following constitutive equations for the direct and converse effects of the piezoelectricity:

$$
\left\{\begin{array}{c}
S_{i}=s_{i j}^{E} T_{j}+d_{k i} E_{k} \\
D_{m}=d_{m j} T_{j}+\varepsilon_{m k}^{T} E_{k}
\end{array}\right.
$$

From those equations we can deduce the charges on the electrodes as:

$$
Q=\int_{A_{E}} D_{3} d A_{E}
$$

where $A_{E}$ is the electrode area and $D_{3}$ the electric displacement in the poling direction (the index 3 indicates the axis of polarization)

Since electric current is the rate of change of charges, measuring the current flowing through the sensor is equivalent to measuring the strain rate directly. However, the current passing through the piezoceramics $\left(I_{P Z T}\right)$ results from the intermingled sensor and actuator signals. It contains the information about the structure's deformation and the piezoelectric actuator's electrical impedance. Thus, it is mandatory to isolate the appearing charges that reflect the deformation of the piezoelectric material $Q_{\delta}$ from those due to the electrical impedance of the piezoelectric actuator $Q_{e}$. We propose a signal conditioning electronics that accurately measures $I_{P Z T}$ and, thanks to a convenient observer retrieves the charges $Q_{\delta}$ generated by the sensor, which is equivalent to measuring its strain. The effects of piezoelectric bending and bending due to external loads are considered separately. The piezoelectric bending results from the contraction or extension in the piezoelectric material enforced by the applied electric field $E$. This is the transduction principle of piezoelectric actuation. For the bending due to external loads, the bender behaves as a passive composite beam. For this paper, we consider the external loads $\left(F_{\text {ext }}\right)$ to be null.

For a one degree of freedom self-sensing implementation, which is the case of this paper, we consider a bilayer unimorph cantilevered beam consisting of an upper piezoelectric layer with polarity $\mathrm{P}$ and a passive lower layer (Figure 2).

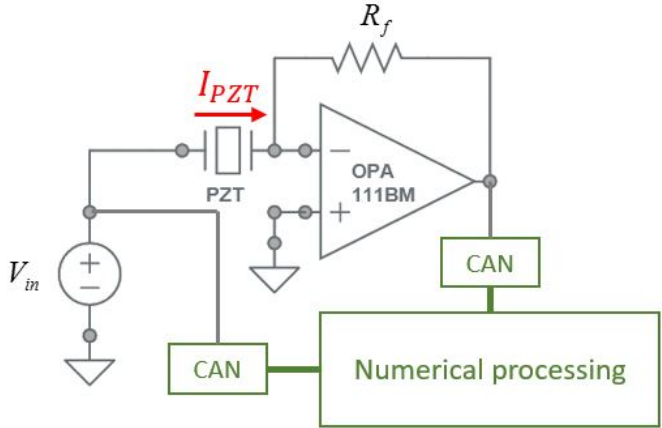

Fig. 3. Electrical schematic of the digital conversion of $I_{P Z T}$.

The current produced by the excitation of the piezoelectric actuator is converted into voltage through a transimpedance (Figure 3). Hence, the relationship between the produced current and the voltage is given by:

$$
V_{\text {out }}=-R_{f}\left(I_{P Z T}+I_{\text {bias }}\right)
$$

where $I_{\text {bias }}$ is the bias current of the used Op. Amp.

From the fundamental equations of the piezoelectric materials (1), the stress $T$ results from the superposition of the mechanical loads and the piezoelectric bending. The part resulting from the piezoelectric extension or contraction is the stress induced by resisting this action [18]. To retrieve the charges that reflect the deformation of the piezoelectric actuator $\int_{A_{E}} d T d A_{E}$ we need to cancel the feedthrough originated by the electrical impedance of the piezoelement $\int_{A_{E}} \varepsilon^{T} E d A_{E}$. As mentioned before, a simple capacitor is sufficient to depict the equivalent electrical impedance of a piezoelement for a given electric field $E$. Hence, the feedthrough current is given by:

$$
I_{e}=C_{r} \frac{d V_{i n}(t)}{d t}
$$

From (3) and (4) we can deduce $Q_{\delta}$ as:

$$
Q_{\delta}(T)=\int_{0}^{T}\left(-\frac{V_{\text {out }}}{R_{f}}-I_{\text {bias }}-C_{r} \frac{d V_{\text {in }}(t)}{d t}\right) d t
$$

which is equivalent to:

$$
Q_{\delta}=\int_{A_{E}}\left(d T+\varepsilon^{T} E-\varepsilon_{r} \frac{V_{i n}}{t \cdot\left(1+S_{3}\right)}\right)
$$

Where $S_{3}$ is the piezoelement strain in the poling direction, $\varepsilon_{r}, C_{r}$ and $t$ represent the piezoelement permittivity, capacitance and thickness in the absence of mechanical deformation and null electric field.

$$
S_{3}=s_{13}^{E} \cdot\left(T_{1}+T_{2}\right)+s_{33}^{E} T_{3}+d_{33} E_{3}
$$

\section{THE NEW OBSERVER}

The developed self-sensing system can be divided into three main parts: the excitation of the piezoelectric actuator with an electric field, the electronic circuit responsible for converting $I_{P Z T}$ into a signal $\left(V_{\text {out }}\right)$ to be used by the observer and, finally, 


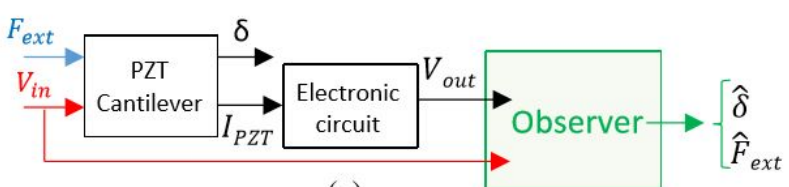

(a)

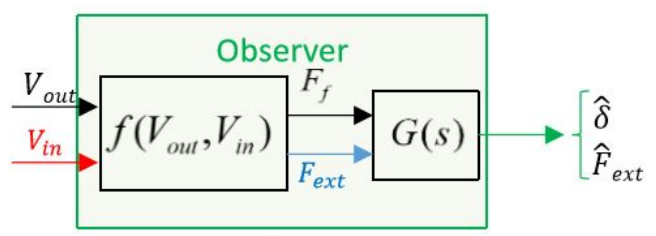

(b)

Fig. 4. Principle of displacement and force observation/estimation using SelfSensing.

the observer that will estimate the displacement $\hat{\delta}$ and the applied force $\hat{F}_{e x t}$ (Figure 4a). With some conditions, it is even possible to use the observer to estimate the internal state (velocity, acceleration) [19]. For this paper, we will focus on estimating the displacement since the external force is null.

The observer is divided into two main parts (Figure $4 \mathrm{~b}$ ) which are described below:

- The first part consists in reconstructing the force responsible for the deformation of the structure. This transduced force $F_{t}$, resulting from the applied electric field $E$, expresses the normal force responsible for the piezoelement extension or contraction in the absence of any external mechanical stress.

- The second part lies in the application of $F_{t}$ to an equivalent mechanical system $G(s)$ that reliably reproduces the mechanical behavior of the piezoelectric actuator. $G(s)$ is supposed to produce the same displacement as the real system when subjected to $V_{i n}$.

\section{EXPERIMENTAL IMPLEMENTATION AND RESULTS}

\section{A. Presentation of the experimental setup}

Figure 5a shows a photo of the piezoactuator. The whole setup, detailed in Figure 5b, is made up of :

- A bilayer unimorph piezoelectric cantilever with dimensions of $20 \mathrm{~mm} \times 2 \mathrm{~mm} \times 0.27 \mathrm{~mm}$, where the thickness of the piezolayer (lead zirconate titanate - PZT - PIC 151) and the thickness of the passive layer (Nickel) are $0.2 \mathrm{~mm}$ and $0.7 \mathrm{~mm}$ respectively,

- A dSPACE-board (DS1103) and a computer for the data acquisition and observer implementation.

- A displacement optical sensor (LC-2420) to measure the displacement at the tip of the actuator. It is set to have some tens of nanometers of resolution and an accuracy of $100 \mathrm{~nm}$,

- A transimpedance "TI" (Figure 3). The used Op. Amp. (OPA111BM) has very low bias currents and noise,

- And a high voltage "HV" amplifier with differential output. It amplifies the voltage $V_{\text {in }}$ from dSPACE.

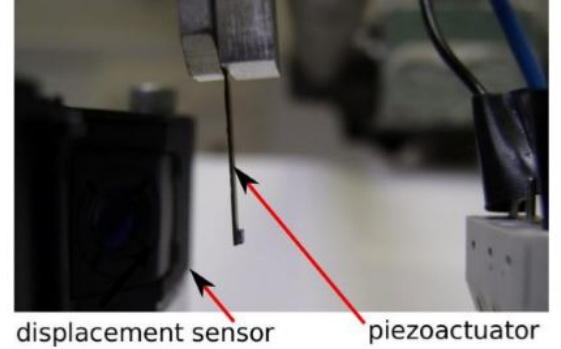

(a)

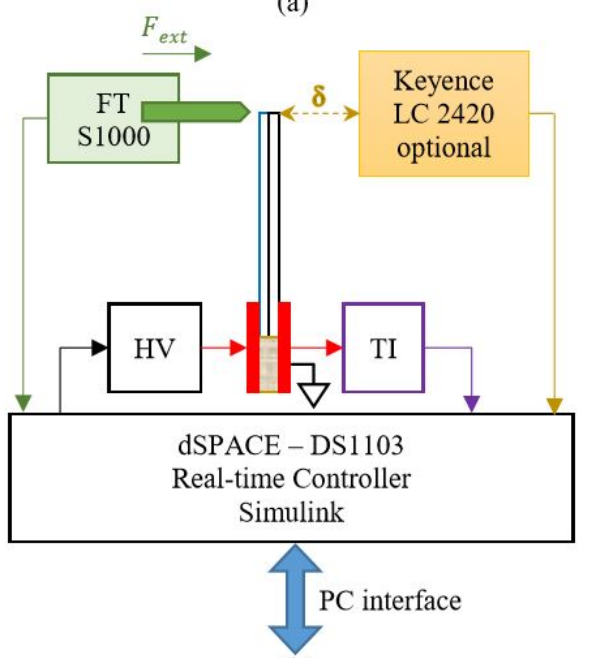

(b)

Fig. 5. (a) Photo of the piezoactuator. (b) Schematic block diagram of the experiment.

\section{B. Observer parameters identification}

To compute the observer, two parameters need to be identified: $F_{t}$ and $G(s)$. Let us first determine $F_{t}$. In a piezoelectric ceramics, there are different regions within the material that have different polar axis and which are called Weiss domains. When subjected to an electric field, each dipole of the piezoelectric remains essentially in its original orientation until the electric field is sufficiently strong to flip it into alignment with the field. The strength of the field required to flip each dipole depends upon its initial orientation. The Flipping of these dipoles will result in the deformation of the piezoelectric ceramics. Its extension or contraction depends on whether the applied voltage $V_{\text {in }}$ has the same polarity as the poling voltage ceramics element. This transduced effect from the electrical to the mechanical domain is described by $F_{t}$. The realignment of the piezoelectric dipoles is responsible for the generation of the deformation charges $Q_{\delta}$. Figure 6 shows the evolution of the produced charges $Q_{\delta}$ and the displacement $\delta$ for several steps.

One can notice two things: after the application of the step, a continuous drift "creep" of the piezoelectric actuator appears both in the displacement and the produced charges. We witness a "transduced direct effect". The term transduced direct effect is used because there is no external mechanical stress applied to generate those charges. Indeed, in this particular case the deformation is due to the continuous realignment of the remaining dipoles that are not yet aligned with the 


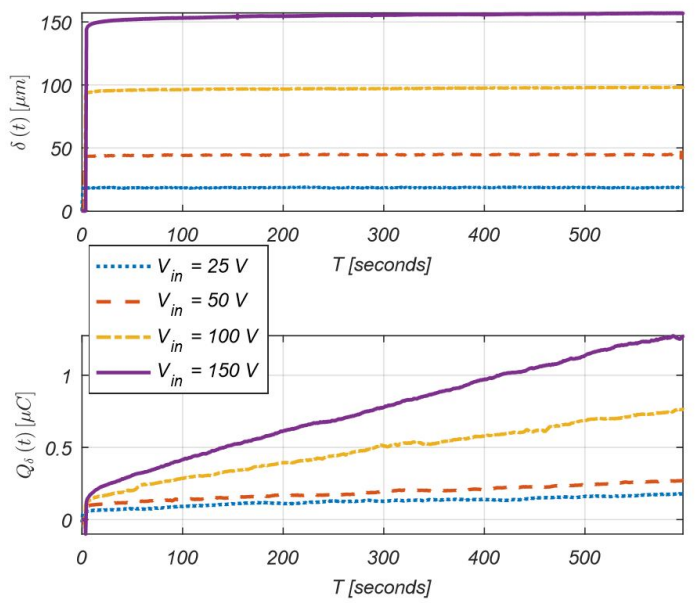

Fig. 6. Several positives Step responses of the piezoelectric actuator: displacement and produced charges.
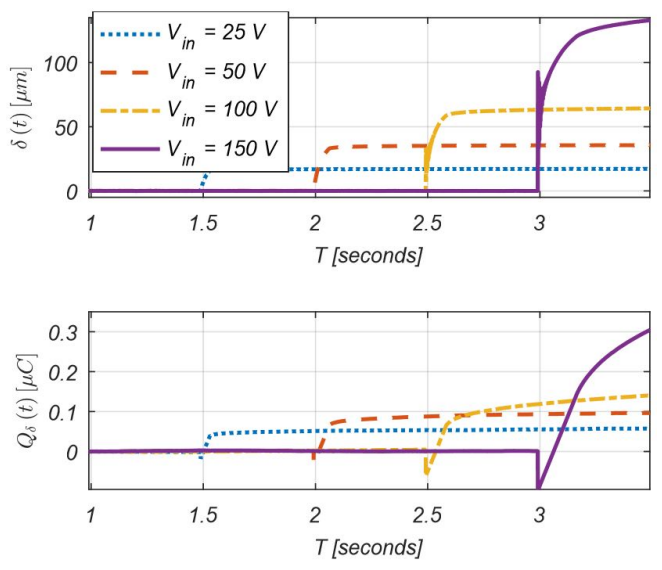

Fig. 7. Close look at the application of the step from Figure 6.

electric field created by $V_{i n}$. The direct effect has an electrical origin and not a mechanical one. Therefore, $Q_{\delta}$ can be used to characterize the transduced force responsible for the creep.

Looking closely to the produced charges at the application of the step (Figure 7), we notice that there is a sudden change in both $Q_{\delta}$ and $\delta$. However, the ratio between the produced charges and the displacement at the application of the step is not equal to the same ratio during the creep:

$$
\left(\frac{Q_{\delta}}{\delta}\right)_{\text {step }} \neq\left(\frac{Q_{\delta}}{\delta}\right)_{\text {creep }}
$$

This makes impossible the use $Q_{\delta}$ to estimate the entire deformation. As a consequence, $Q_{\delta}$ can be used to express either the deformation during the step or the creep. Furthermore, the change in $Q_{\delta}$ during the step remains proportional to $V_{\text {in }}$ (Table I). Indeed, due to the sudden application of the electric field, the piezoelectric actuator is deformed and its capacitance changed.

Up to now, we assumed that the charges due to the transduced direct effect $Q_{\delta}$ could be calculated with equation (5) or (6). This assumption is applicable for low electric field only. Indeed, piezoelectric coefficients $\left(d_{33}, \varepsilon\right)$ show a strong electric field dependency [20,21].

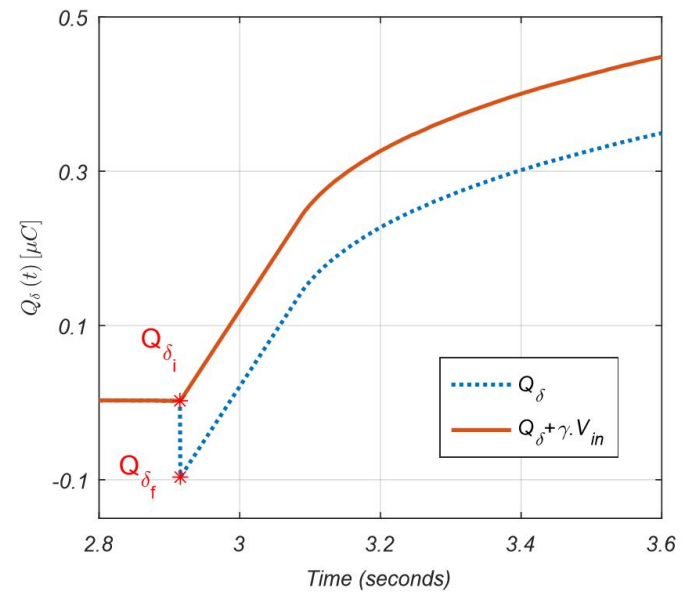

Fig. 8. Produced charges $Q_{\delta}$ taking into account the dynamical variation of the impedance of the piezoelectric actuator.

Instead of an analytical model to depict the piezoelectric coefficients electric field dependency for a thorough feedthrough cancelation, we opted for an empirical parameter to represent all the parameters changes during the piezoelectric actuator deformation. We introduce the term $\gamma$ that captures this change in the piezoelectric capacitance and that is used to take into account the variation of the piezoelectric actuator impedance. Hence, $\gamma$ is included in the determination of $F_{t}$ to suppress the impact of the sudden change of the piezoelectric actuator impedance in the produced charges. $\gamma$ updates the piezoelement's capacitance and eliminates the induced change at the moment the step is applied in the produced charges (Figure 8).

Since $Q_{\delta}$ cannot be used to depict both the creep and the change at the step application, the produced charges corrected from the piezoelement impedance change $Q_{\delta}+\gamma \cdot V_{i n}$ is used o depict the force responsible for the creep and $V_{i n}$ is used to determine the force causing the deformation at the step application. As a result, the transduced force is defined as:

$$
F_{t}=\alpha \cdot V_{i n}+\beta\left(Q_{\delta}+\gamma \cdot V_{i n}\right)
$$

Where $\alpha$ and $\beta$ are defined by :

$$
\begin{gathered}
\alpha=\frac{\delta_{s s}}{\left(V_{i n}\right)_{t \rightarrow} \cdot G(0)} \\
\alpha=\frac{e_{\delta}}{e_{Q_{\delta}} \cdot G(0)}
\end{gathered}
$$

where $\delta_{s s}$ is the displacement of the tip of the piezoelectric actuator at the end of the transient part, $e_{\delta}$ and $e_{Q_{\delta}}$ are respectively the measured "creep" in the displacement and the produced charges (Figure 9) and, $G(0)$ is the steady state gain of the system $G(s)$.

To determine $\alpha$ and $\beta$, various positive steps are applied to the PZT actuator. Then, data after the transient part are used on equations (10) and (11). We identified $\alpha$ and $\beta$ as:

$$
\alpha=0.8971
$$




$$
\beta=6.9067 \times 10^{7}
$$

The missing part that will permit to completely define the observer is the vibrational dynamics of the mechanical structure of the piezoelectric actuator. In general, the vibration modeling of the mechanical structure consists on the development of a linear vibrational dynamics models for the piezoactuated stages without considering the hysteresis nonlinearity $[5,22]$. In this sense, the input and output data of the piezo actuated stages can be directly used to obtain the linear dynamics models or the transfer function using a commercial dynamic signal analyzer or the system identification toolbox in matlab. Figure 9 shows a step response of the used piezoelectric actuator: the displacement and the produced charges during and after the transient part. The identification of $G(s)$ is performed by applying a step voltage $V_{i n}$ to the actuator without force at the tip and then by capturing the output $\delta$ thanks to the optical sensor. Finally, the ARMAX (Auto Regressive Model with eXternal input) method is applied to the captured data of the transient part [23]. Through this method, we identified a third order transfer function that fits the measured data at $88.69 \%$.

TABLE I

PRODUCED CHARGES AT THE APPLICATION OF THE STEP AND THE CORRECTION TERM OF THE IMPEDANCE OF THE PZT ACTUATOR $\gamma($ FROM FIGURE 8)

\begin{tabular}{|c|c|c|c|}
\hline$V_{\text {in }}[V]$ & $Q_{\delta_{i}}[n C]$ & $Q_{\delta_{f}}[n C]$ & $\gamma=\frac{\left(Q_{\delta_{i}}-Q_{\delta_{f}}\right)}{V_{\text {in }}}[n F]$ \\
\hline 25 & -2.67 & -19.16 & 0.66 \\
\hline 50 & 4.06 & -28.89 & 0.66 \\
\hline 100 & 8.32 & -57.65 & 0.66 \\
\hline 150 & 1.93 & -97.07 & 0.66 \\
\hline
\end{tabular}

\section{Experimental verification of the efficiency of the self- sensing}

Figure 10 compares the measured displacement $\delta_{\text {meas }}$ at the tip of the piezoelectric actuator and the estimated displacement $\delta_{\text {est }}$ through the suggested self-sensing with and without $\gamma$, the corrective term of the dynamical impedance of the PZT actuator. Let us analyze Figure 10 and highlight the main difference between self-sensing on previous approaches and the contribution brought by this paper. On previous works [25, $26]$ the main assumption was that, for an input voltage $V_{i n}$, the piezoelectric cantilever actuator is bent with deflection $\delta$. If this bending is free, i.e. no external mechanical stress applied to the actuator, the charges that appear on the electrode of the actuator are expressed as $Q=C_{r} \cdot V_{i n}+Q_{\delta}$. Moreover, since the produced charges are quasi-proportional to free displacement, it was assumed that $Q_{\delta}=\beta_{2} \cdot \delta$ [27], where $\beta_{2}$ is the charge-displacment coefficient. Because $C_{r}$ is constant, $Q_{\delta}$ is obtained thanks to a static feedthrough cancellation. As the piezoactuator's impedance variation is not accounted for, this model is limited to few tens of volts, where the change in the impedance is almost imperceptible due to small electric fields. The creep, which is electric field dependent

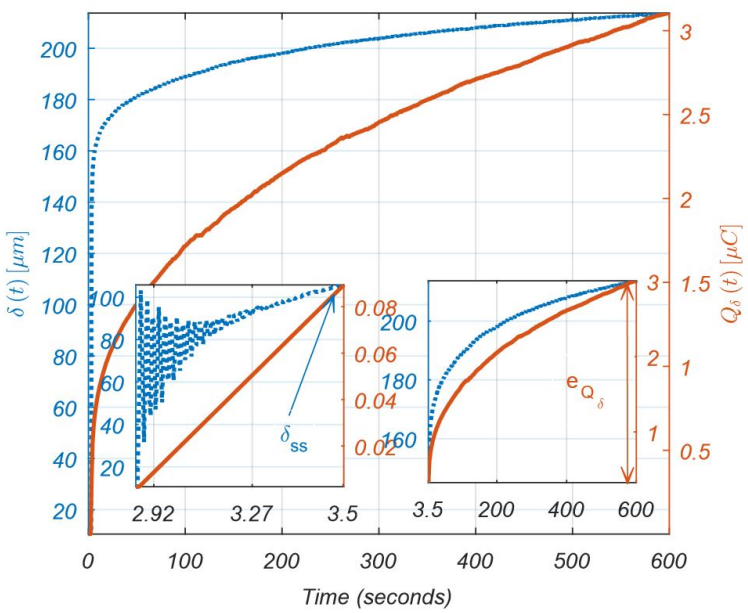

Fig. 9. Step response $\left(V_{\text {in }}=200 V\right): \delta$ and $Q_{\delta}$ (overall, transient part and Creep.

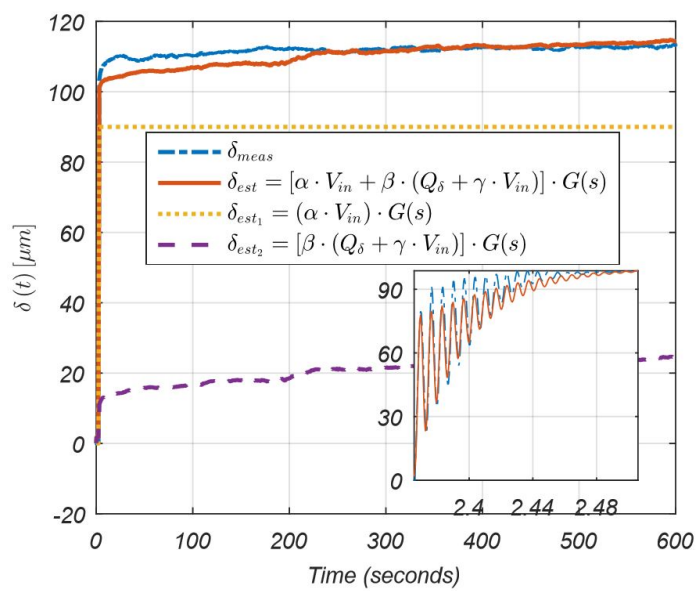

Fig. 10. Measured and estimated displacement with and without the correction term $\gamma$ for a constant step of $V_{i n}=100 \mathrm{~V}$

(Figure 6), makes possible the determination of $\beta_{2}$ for weak electric field such that $\delta_{\text {est }} \rightarrow \delta_{\text {meas }}$. This is not possible for hundreds of volts where the piezoactuator's impedance change is more pronounced (Figure 7) and principally because of the consideration made in (8).

For $V_{i n}=100 \mathrm{~V}$, we notice that the estimated displacement using the proportional relationship between produced charges and displacement $\delta_{\text {est }}$ is far from the measurement realized with the optical sensor $\delta_{\text {meas }}$. However, using the transduced force $F_{t}$ as defined in (9), the observer was able to track the output displacement of the actuator for both the transient and the creep part (Figure 10). These results clearly show that the used observer achieves very good precision when working with a long-term constant condition with an excellent approximation of the dynamics of the actuator. One can see that, despite a slight difference between the measured displacement and the predicted behavior for the first 200 seconds, the estimation error tends to zero and remains within an interval of $\pm 1 \%$ of the desired displacement.

Furthermore, the transient part of the estimated displacement presents a minor difference compared to the real dis- 
placement. It is worth to remind that the accuracy of the estimated signal from the self-sensing and its observer relative to the real displacement is strongly dependent on the precision of the identified model $G(s)$. Since, the identified $G(s)$ has a fitting confidence parameter of $88.69 \%$ compared to the experimental data, it is normal to notice this slight difference between both.

This paper proposes a self-sensing measurement technique devoted to estimate the free displacement of the tip of a unimorph piezoelectric actuator with consideration of the impact of its deformation to its electrical impedance. Relative to existing self-sensing works that are limited to small displacement and low electric field, the suggested self- sensing approach is able to estimate large displacements in piezoactuator. Furthermore, the use of a black box to describe the dynamics of the piezoactuator permits a better approximation of the behavior of the system. Indeed, with a black box we were able to enlarge the working frequency range and thus to capture all the antiresonances and resonances for a given frequency range depending on the complexity/order of the black box transfer function.

\section{CONCLUSION AND PERSPECTIVES}

We present a new self-sensing method and related observer to estimate the displacement at the tip of cantilevered piezoelectric based actuators. The novelty in the proposed approach is the consideration of the impact of the mechanical deformation of the piezoelectric element on its electrical impedance. A numerical cancellation of the feedthrough generated by the piezoelectric actuator electrical impedance is realized for a more accurate measurement of the charges that reflects its deformation. Compensation of the dynamical behavior of the electrical impedance of the piezoelement is performed through an observer in order to improve the estimator's performances.

The observer has been validated for a one degree of freedom (1-DoF) unimorph cantilevered actuator displacement estimation. The next steps are, in addition to displacement, the estimation of external stresses and the development of a self-sensing based observer for a 3-axis actuator of both displacement and force of uni- and bimorph piezoelectric based actuator.

\section{REFERENCES}

[1] M. Rakotondrabe, "Smart materials-based actuators at the micro/nanoscale: characterization, control and applications," in Springer Verlag, New York, 2013.

[2] C. Lee and S. M. Salapaka, "Fast Robust Nanopositioning?A LinearMatrix-Inequalities-Based Optimal Control Approach.," IEEE/ASME Trans. on Mechatronics, vol. 14, no. 4, pp. 414-422, 2009.

[3] D. Habineza, M. Rakotondrabe and Y. Gorrec, "Bouc- Wen Modeling and Feedforward Control of Multivariable Hysteresis in Piezoelectric Systems: Applocation to a 3-DoF Piezotube Scanner," IEEE Trans. on Control Systems Technology, pp. 1-10, 2015.

[4] Y. Zhang and P. Yan, "Modeling, identification and compensation of hysteresis nonlinearity for a piezoelectric nano-manipulator," Journal of Intelligent Material Systems and Structures , pp. 1-16, 2016.

[5] M. Rakotondrabe, I. A. Ivan, S. Khadraoui, P. Lutz and N. Chaillet, "Simultaneous Displacement/Force Self- Sensing in Piezoelectric Actuators and Applications to Robust Control," IEEE/ASME Trans. on Mechatronics, vol. 20, no. 2, pp. 519-531, 2015.
[6] V. Hofmann and J. Twiefel, "Self-Sensing with Loaded Piezoelectric Bending Actuators," Sensors and Actuators: A Physicalhttp://dx.doi.org/10.1016/j.sna.2017.06.004.

[7] J. J. Dosch, D. J. Inman and E. Garcia, "A self-Sensing Piezoelectric Actuator for Collocated Control," Journal of Intelligent Material Systems and Structures, vol. 3, pp. 166-185, 1992.

[8] Inman and D. J., "Control/Structure Interaction: Effects of Actuator Dynamics.," Mechanics and Control of Large Flexible Structures, Progress in Astronautics and Aeronautics., vol. 129, pp. 507-533, 1990.

[9] W. W. Law, W.-H. Liao and J. Huang, "Vibration control of structures with self-sensing piezoelectric actuators incorporating adaptive mechanisms.," Smart materials and structures, vol. 12, pp. 720-730, 2003.

[10] M. G. Ruppert and S. O. R. Moheimani, "High- bandwidth multimode self-sensing in bimodal atomic force microscopy.," Beilstein Journal of Nanotechnology, vol. 7, pp. 284-295, 2016.

[11] M. Sasaki and S. Ito, "Vibration Control of a Micro-Actuator for the Hard Disk Drive using Self-Sensing Actuation," in Int. Conf. on Control, Automation and Systems, Seoul, Korea, 2008.

[12] K. Seki and M. Iwasaki, "Application of Self-sensing Technique for Position Control Considering Vibration Suppression in Piezo-driven Stage.," in IEEE Int. Conf. on Mechatronics (ICM), Nagoya, Japan, 2015.

[13] K. Seki and M. Iwasaki, "Improvement of Bending Vibration Suppression Performance for Galvano Mirror by Self-Sensing Actuation.," IEEJ Journal of Industry Applications, vol. 3, no. 1, pp. 10-17, 2014.

[14] I. A. Ivan, M. Rakotondrabe, P. Lutz and N. Chaillet, "Quasistatic displacment self-sensing method for cantilevered piezoelectic actuators," Review of Scientific Instruments, vol. 6, pp. 1-8, 2009.

[15] M. Goldfarb and N. Celanovic, "A lumped parameter electromechanical model for describing the nonlinear behavior of piezoelectric actuators," ASME Journal of Dynamic Systems, Measurement, and Control, vol. 119, pp. 478-485, 1997.

[16] C. H. Park, "On the circuit model of piezoceramics," Journal of Intelligent Material Systems and Structures, vol. 12, pp. 2354-2363, 2001.

[17] G. Mingjie and W.-H. Liao, "Studies on the Circuit Models of Piezoelectric Ceramics," in Int. Conf. on Information Acquisition, Hefei, China, 2004.

[18] R. Dunsch and J.-M. Breguet, "Unified mechanical approach to piezoelectric bender modeling," Sensors and Actuators A 134, pp. 436-446, 2006.

[19] M. Rakotondrabe, "Combining self-sensing with an Unkown-InputObserver to estimate the displacement, the force and the state in piezoelectric cantilevered actuator," in American Control Conf., Washington USA, 2013.

[20] H. Ikeda and T. Morita, "High-precision positioning using a self-sensing piezoelectric actuator control with a differential detection method," Sensors and Actuators A 170, pp. 147-155, 2011.

[21] M. Quant, H. Elizalde, A. Flores, R. Ramirez, P. Orta and G. Song, "A comprehensive model for piezoceramic actuators: modelling, validation and application," Smart Materials and Structures, vol. 18, no. 12, 2009.

[22] G. M. Clayton, S. Tien, K. K. Leang , Q. Zou and S. Devasia, "A review of feedforward control approaches in nanopositioning for highspeed SPM.,' J. Dynam. Syst., Meas., Control, , vol. 131, no. 16, pp. 61-101, 2009.

[23] L. Ljung, System Identification: Theory for the User, Upper Saddle, NJ: Prentice-Hall PTR, 1999.

[24] I. Ivan , O. Aljanaideh, J. Agnus, P. Lutz and M. Rakotondrabe, ”Quasistatic displacement self-sensing measurement for a 2-DOF piezoelectric cantilevered actuator,' IEEE/ASME Trans. on Industrial Electronics, pp. 1-8, 2017.

[25] M. N. Islam and R. J. Seethaler, "Sensorless Position Control For Piezoelectric Actuators Using A Hybrid Position Observer," IEEE/ASME Trans. on Mechatronics, vol. 19, no. 2, pp. 667-675, 2014.

[26] G.-Y. Gu, L.-M. Zhu, C.-Y. Su, H. Ding and S. Fatikow, "Modeling and Control of Piezo-Actuated Nanopositioning Stages: A Survey," IEEE Trans. on Automation Science and Engineering, vol. 13, no. 1, pp. 313$332,2016$. 